\title{
Sucrose degradation is regulated by 1-methycyclopropene treatment and is related to chilling tolerance in two peach cultivars
}

Lina Yu, Xingfeng Shao *, Yingying Wei, Feng Xu, Hongfei Wang

Department of Food Science and Engineering, Ningbo University, Ningbo 315211, PR

China

*Corresponding author. Tel.: +86-574 87609573; Fax: +86-574 87608347;

E-mail address: shaoxingfeng@nbu.edu.cn (X. Shao). 


\section{ABSTRACT}

In order to reveal how sucrose degradation is related to chilling tolerance, two varieties of peach fruit, 'Zajiao' and 'Yulu', were treated with 1-methylcyclopropene (1-MCP) vapor at $1 \mu \mathrm{L} / \mathrm{L}$ for $24 \mathrm{~h}$ before storage at $5{ }^{\circ} \mathrm{C}$ for 35 and $28 \mathrm{~d}$ respectively. 'Yulu' peaches had higher sucrose content than 'Zajiao' peaches at harvest, but a higher rate of sucrose degradation in untreated 'Yulu' fruit may have resulted greater sensitivity to chilling stress compared with untreated 'Zajiao' fruit. 1-MCP treatment significantly inhibited chilling injury $(\mathrm{CI})$ and preserved higher firmness values in both varieties. This treatment reduced the activities and expression of enzymes related to sucrose degradation, and increased activity and expression of sucrose synthase synthesis, resulting in a lower rate of sucrose degradation and the increase of glucose and fructose content in both varieties during cold storage. These results suggest that lower degradation rates of sucrose during cold stress, rather than the higher content of sucrose at harvest time, enhances chilling tolerance in peach fruit.

Keywords: Prunus persica, 1-methylcyclopropene, chilling injury, sucrose, sugar metabolism 


\section{Introduction}

Peaches are sought by consumers around the world for their good taste and high nutritional value. Although peach fruit are climacteric and have a short shelf life when stored at ambient temperature, cold storage is a practical method to slow ripening (Cantín et al., 2010). However, the storage temperature is critical, and peaches are susceptible at $5{ }^{\circ} \mathrm{C}$, but not at $0{ }^{\circ} \mathrm{C}$ or $10{ }^{\circ} \mathrm{C}$ (Wang et al., 2013; Yu et al., 2015) to chilling injury $(\mathrm{CI})$, manifested by internal browning, flesh mealiness, and loss of flavor(Lurie and Crisosto, 2005). These symptoms can be reduced by hot air (Wang et al., 2014), methyl jasmonate (Jin et al., 2009), and oxalic acid (Jin et al., 2014).

The greatest capacity of the peach/nectarine fruit to produce ethylene after cold storage was associated to lower CI (Giné-Bordonaba et al., 2016). Maintaining the ability to produce ethylene or adding exogenous ethylene can prevent CI of nectarine fruit (Zhou et al., 2001). 1-Methylcyclopropene (1-MCP) is an ethylene perception inhibitor that is widely used to reduce ethylene production, slow the ripening process and prolong shelf life in horticultural crops (Watkins, 2006). Interestingly, studies have confirmed that 1-MCP treatment has inhibitive effects on CI of loquat (Cao et al., 2009), pear (Cheng et al., 2015), persimmon (Salvador et al., 2004) and citrus fruit (Dou et al., 2005). Puig et al. (2015) demonstrated that although ethylene is related to tolerance to cold storage of peach, differential auxin subcellular accumulation and signaling may play a role in determining chilling sensitivity/tolerance. This maybe the reason that 1-MCP inhibits the release of ethylene but not resulted in the reduction of chilling tolerance in some fruits. However, the effect of 1-MCP treatment on CI of 
peaches and nectarines is contrary. Jin et al. (2011) reported that $0.5 \mu \mathrm{L} / \mathrm{L} 1-\mathrm{MCP}$ treatment retarded internal browning and flesh mealiness in 'Baifeng' peaches. The CI index was also inhibited by two doses $(0.5$ and $1 \mu \mathrm{L} / \mathrm{L})$ of $1-\mathrm{MCP}$ in nectarines (Özkaya et al., 2016). In contrast, other researchers found that treatments with 0.5 $\mu \mathrm{L} / \mathrm{L}$ or $0.9 \mu \mathrm{L} / \mathrm{L} 1-\mathrm{MCP}$ increased CI symptoms in 'Elberta' or 'Chiripá' peaches (Fan et al., 2002; Girardi et al., 2005). These contrary results maybe related with the different fruit cultivars and 1-MCP concentration.

Sucrose and reducing sugars (glucose and fructose) are the major soluble sugars in peach fruit at harvest. The dominant sugar is sucrose, representing about $75 \%$ of total sugar (Aubert et al., 2014). Soluble sugars are closely linked to flavor and nutritional value, and also help support resistance to low-temperature and other osmotic stresses by regulating osmotic pressure, protecting cell membranes from fusion and leakage (van den Bogaart et al., 2007), providing oxidation resistance (Bolouri-Moghaddam et al., 2010) and controlling gene expression (Wang et al., 2013). Recently, soluble sugars have been shown to reduce CI development of postharvest fruits during cold storage. Higher levels of reducing sugars improve CI tolerance in loquat (Cao et al., 2013; Shao et al., 2013) and apricot fruit (Wang et al., 2016). However, in other fruit such as peach (Wang et al., 2013) and mandarin (Holland et al., 2002), sucrose, rather than reducing sugars, is related to enhanced CI tolerance. Our previous study showed that chilling stress stimulates the activities and transcription levels of enzymes involved in sucrose metabolism, resulting in increased sucrose cleavage in peach fruit (Yu et al., 2015). The increased sucrose levels that 
occur during cold storage enhance the chilling tolerance in peach fruit treated with hot air and methyl jasmonate (MeJA) (Yu et al., 2016).

The ability of 1-MCP to alleviate CI in peach fruit has been attributed to antioxidant enzyme activities (Jin et al., 2011). 1-MCP treatment reduced pectin methylesterase and polygalacturonase activities, leading to lower CI incidence in nectarine fruit (Özkaya et al., 2016). Similar observations have been reported in other fruits treated with 1-MCP, such as pears (Cheng et al., 2015), persimmon (Salvador et al., 2004) and loquat (Cao et al., 2009). However, the relationship between 1-MCP treatment and soluble sugar metabolism has not been explored. To examine sucrose degradation and the development of CI during cold storage $\left(5^{\circ} \mathrm{C}\right)$, we treated two varieties of peach fruit with1-MCP and monitored CI index, soluble sugar content, soluble sugar metabolism-related enzyme activity, and gene expression.

\section{Materials and methods}

\subsection{Plant material and experimental design}

1-MCP formulation (Lubo Fresh) was purchased from Xinyi Institute of Fruit and Vegetable Quality, Jiangsu Province, China. 'Zajiao' and 'Yulu' peach fruits (Prunus persica L. Batsch) were harvested at optimum commercial maturity (about $80 \%$ maturity levels, accordance to growers recommendations) from a commercial orchard in Fenghua, Zhejiang Province, China. After harvest, the fruits were immediately transported to the laboratory and precooled at $5{ }^{\circ} \mathrm{C}$ for $3 \mathrm{~h}$ to remove field heat. Fruits were selected for uniform size and shape and lack of physical or mechanical damage. 
Peach fruits from each cultivar were randomly assigned to 1-MCP treated or control groups. Each group consisted 270 fruits divided into three biological replicates. The experimental group was treated with $1 \mu \mathrm{L} / \mathrm{L} 1-\mathrm{MCP}$ vapor in a sealed container for 24 $\mathrm{h}$ at $5{ }^{\circ} \mathrm{C}$. After treatment, the container was opened and ventilated for $1 \mathrm{~h}$, the 'Zajiao' peaches were stored at $5{ }^{\circ} \mathrm{C}$ for $35 \mathrm{~d}$, the 'Yulu' peaches, were stored at $5{ }^{\circ} \mathrm{C}$ for $28 \mathrm{~d}$. The untreated control peaches were stored at $5{ }^{\circ} \mathrm{C}$; $35 \mathrm{~d}$ for 'Zajiao', $28 \mathrm{~d}$ for 'Yulu'. Fifteen fruits per replicate were analyzed at 7-day intervals during cold storage. CI indexes and firmness were assessed immediately after removal from cold storage, and then peaches were pooled and immediately frozen in liquid nitrogen. All frozen samples were stored at $-40{ }^{\circ} \mathrm{C}$ for further analysis.

\subsection{Determination of $C I$ index and firmness}

Internal browning is a frequent postharvest disorder that can be observed when peach and nectarine fruits are maintained in cold storage for long periods (Cáceres et al., 2016). Thus, internal browning was estimated visually to calculate as the CI index, using the brown area observed after cutting each fruit along its axial diameter (Wang et al., 2013). CI level was graded using the following scale: $0=$ none; $1=$ browning area less than $5 \% ; 2=$ browning area between $5 \%$ and $25 \% ; 3=$ browning area between $25 \%$ and $50 \% ; 4=$ browning area more than $50 \%$. CI index was calculated as $[(\mathrm{CI}$ level $) \times($ the number of fruits at this $\mathrm{CI}$ level $)] /(4 \times$ total number of fruits in each treatment).

Firmness was determined using a digital fruit hardness tester (GY-4, Dongwan 
City Zhiqu Precision Instrument Co., Ltd, China) fitted with an $8 \mathrm{~mm}$ diameter probe. The puncture distance was $10 \mathrm{~mm}$. Firmness was measured in Newtons $(\mathrm{N})$.

\subsection{Measurement of soluble sugars}

Soluble sugar content was analyzed using the method described byShao et al. (2013). $5 \mathrm{~g}$ samples of frozen peach tissue were finely ground in a solution containing $0.5 \mathrm{ml} 300 \mathrm{mM}$ zinc acetate and $0.5 \mathrm{ml} 63 \mathrm{mM}$ potassium ferrocyanide. The homogenate was diluted to $25 \mathrm{ml}$ with deionized water and then passed through a 0.22 $\mu \mathrm{m}$ membrane filter. Soluble sugars were measured using a high performance liquid chromatography (HPLC) system (Model 2695, Waters, USA), an XBrige ${ }^{\mathrm{TM}}$ Amide Column $(3.5 \mu \mathrm{m}, 4.6 \times 250 \mathrm{~mm}$, USA), and a refractive index (RI) detector (Model 2414, Waters, USA). A $20 \mu \mathrm{L}$ aliquot was injected into the HPLC system for analysis. The mobile phase composition was acetonitrile/water $(80: 20$, v/v). The total flow rate was $1 \mathrm{ml} \mathrm{min}^{-1}$ and the column temperature was $35^{\circ} \mathrm{C}$.

\subsection{Extraction and assays of sucrose metabolism-related enzyme activities}

Enzymes were extracted using the method of Zhang et al. (2012). Frozen peach tissue $(1 \mathrm{~g})$ was homogenized on ice in $5 \mathrm{ml}$ buffer containing $100 \mathrm{mM}$ sodium phosphate ( $\mathrm{pH} 7.5), 5 \mathrm{mM} \mathrm{MgCl} 2,1 \mathrm{mMEthylenediaminetetraacetic} \mathrm{acid} \mathrm{(EDTA),} 2.5$ $\mathrm{mM}$ dithiothreitol (DTT), and $0.1 \%(\mathrm{v} / \mathrm{v})$ Triton X-100. The homogenates were centrifuged at $10,000 \times \mathrm{g}$ for $20 \mathrm{~min}$ at $4{ }^{\circ} \mathrm{C}$. The supernatant was dialyzed immediately in a 10-fold volume of extraction buffer (without Triton X-100) at $4{ }^{\circ} \mathrm{C}$ 
for $20 \mathrm{~h}$. The crude enzyme extracts were used for measurements of the following enzyme activities.

Acid invertase (AI) activity was assayed using the method of Sun et al. (2011). The reaction consisted of $100 \mathrm{mM}$ sodium citrate buffer $(\mathrm{pH} 4.5), 1 \%(\mathrm{~m} / \mathrm{v})$ sucrose, and crude enzyme extract. The mixture was incubated for $30 \mathrm{~min}$ at $37{ }^{\circ} \mathrm{C}$. 3 , 5-dinitrosalicylic acid was then added and the sample placed in a boiling water bath for 5 min to terminate the reaction. Absorbance at $540 \mathrm{~nm}$ was measured after cooling. Neutral invertase (NI) activity was assayed similarly, but using $100 \mathrm{mM}$ sodium phosphate buffer ( $\mathrm{pH}$ 7.5). Sucrose synthase-cleavage (SS-cleavage) activity was assayed in a mixture of $80 \mathrm{mM}$ Hepes- $\mathrm{NaOH}(\mathrm{pH}$ 5.5), $5 \mathrm{mM} \mathrm{NaF}, 5 \mathrm{mM}$ uridinediphosphate (UDP), $100 \mathrm{mM}$ sucrose and crude enzyme extract. Subsequent steps were as described for AI. AI, NI and SS-cleavage activities were expressed as micromoles glucose per hour per gram fresh weight (FW).

SS-synthesis and sucrose phosphate synthase (SPS) activities were assayed using the method of Solomakhin and Blanke (2010). The SS-synthesis assay consisted of $100 \mathrm{mM}$ Hepes- $\mathrm{NaOH}$ (pH 8.0), 4mM uridinediphosphate glucose, $15 \mathrm{mM} \mathrm{MgCl}_{2}, 60$ $\mathrm{mM}$ fructose and crude enzyme extract. The SPS assay consisted of $100 \mathrm{mM}$ HEPES-NaOH (pH 8.0), $10 \mathrm{mM}$ UDP-glucose, $5 \mathrm{mM}$ fructose-6-phosphate, $15 \mathrm{mM}$ glucose-6-phosphate, $15 \mathrm{mM} \mathrm{MgCl}$ and crude enzyme extract. The mixture was incubated for $30 \mathrm{~min}$ at $37^{\circ} \mathrm{C}$. The reaction was terminated by adding $5 \mathrm{mM} \mathrm{NaOH}$ and then placing the sample in boiling water for $10 \mathrm{~min}$. After cooling, anthrone was added to the mixture and the sample was incubated for $10 \mathrm{~min}$ at $80{ }^{\circ} \mathrm{C}$. The samples 
were allowed to cool, and absorbance was measured at $620 \mathrm{~nm}$. SS synthesis and SPS activities were expressed as micromoles sucrose per hour per gram $\mathrm{FW}$.

\subsection{RNA isolation and real-time PCR analysis}

Following the method of (Meisel et al., 2005), $2 \mathrm{~g}$ frozen peach tissue was thoroughly ground in liquid nitrogen then suspended in a solution of hexadecyltrimethyammonium bromide (CTAB). The mixture was incubated for 20 $\min$ at $65{ }^{\circ} \mathrm{C}$. After incubation, the solution was mixed with chloroform : isoamyl alcohol $(24: 1)$ then centrifuged at $12,000 \times \mathrm{g}$ for $35 \mathrm{~min}$ at $4{ }^{\circ} \mathrm{C}$. The supernatant was mixed with $\mathrm{LiCl}$ and incubated overnight at $4{ }^{\circ} \mathrm{C}$. Samples were centrifuged at 12,000 $\times \mathrm{g}$ for $35 \mathrm{~min}$ at $4{ }^{\circ} \mathrm{C}$. The sediment was dissolved in a solution of SSTE and chloroform : isoamyl alcohol (24:1), and then centrifuged at $14,000 \times \mathrm{g}$ for $10 \mathrm{~min}$ at $4{ }^{\circ} \mathrm{C}$. The supernatant was mixed with a 2 -fold volume of absolute ethyl alcohol and chilled at $-80{ }^{\circ} \mathrm{C}$ for $30 \mathrm{~min}$. The homogenate was centrifuged again at $14,000 \times \mathrm{g}$ for 20 min at $4{ }^{\circ} \mathrm{C}$. The sediment was washed with $70 \%$ ethyl alcohol and centrifuged at $14,000 \times \mathrm{g}$ for $10 \mathrm{~min}$ at $4{ }^{\circ} \mathrm{C}$, and then allowed to dry in a clean environment. According to the manufacturer's instructions, samples were treated with RNase-free DNase (TaKaRa, Japan) to eliminate genomic DNA. RNA sample integrity was evaluated by electrophoresis using a $1 \%$ agarose gel. The concentration of DNA-free RNA was determined using a spectrophotometer (NanoDrop 1000, Thermal, USA). RNA samples were stored at $-80^{\circ} \mathrm{C}$.

Following the manufacturer's protocol, first-strand cDNA was synthesized from 
$2 \mu \mathrm{g}$ of treated total RNA using the SYBR PrimeScript RT-PCR KitII reverse transcriptase (TaKaRa, Japan). The cDNA was diluted 10-fold with DEPC-treated water and stored at $-20^{\circ} \mathrm{C}$ until analysis by Quantitative real-time PCR (RT-qPCR).

RT-qPCR was conducted to determine relative gene expression levels, using the SYBR Green Kit II (TaKaRa, Japan). RT-qPCR primers sequences and accession numbers were designed same as Yu et al. (2016). PCR reactions were carried out according to the manufacturer's instructions. Relative gene expression was calculated by the 'Comparative $2^{-\Delta \Delta \mathrm{CT}}$ method (Livak and Schmittgen, 2001). Translation elongation factor 2 (TEF2, JQ732180.1) was used as an internal control (Wang et al., 2013), and cycle threshold (Ct) numbers were obtained for both the reference and target genes. The results were expressed according to the method of Thomsen et al. (2010). Each RNA sample was run in triplicate.

\subsection{Statistical analysis}

Experiments were performed using a randomized design. All statistical analyses were performed with SAS (version 8.2, SAS Institute, Cary, NC, USA). Mean separations were compared using Duncan's multiple range tests with a significance threshold of 0.05. Figures were prepared using Origin Pro 8.1 G (Microcal Software Inc., Northampton, MA, USA).

\section{Results}

\subsection{Fruit firmness and CI index}


Although pulp firmness decreased during cold storage in both varieties of treated and untreated peaches, the 1-MCP treated groups maintained significantly $(p<0.05)$ greater firmness (Fig. $1 \mathrm{~A}$ and C). CI was characterized mainly by the appearance of flesh browning. 'Zajiao' peach fruit exhibited visible CI symptoms on the 28th day of cold storage (Fig. $1 \mathrm{~B}$ ), and CI in 'Yulu' peach fruit was first observed after $21 \mathrm{~d}$ (Fig. $1 \mathrm{D})$, becoming more pronounced on day 28 . After $28 \mathrm{~d}$ of storage, the CI index of 'Yulu' control peaches was 0.43 , and of 'Zajiao' control peaches was 0.35 . Hence, 'Zajiao' fruit showed naturally higher chilling tolerance than 'Yulu' fruit. 1-MCP treatment significantly reduced CI indexes. For 'Zajiao' peach fruit at days 28 and 35, 1-MCP treatment reduced CI $51.43 \%$ and $46.59 \%$ respectively, compared to control peaches (Fig.1 B). For 'Yulu' peach fruit at 21 and $28 \mathrm{~d}$, CI was reduced $73.91 \%$ and 81.40\% respectively, compared to controls (Fig. 1 D).

\subsection{Changes in sucrose, glucose, and fructose content}

Throughout the experiment, sucrose content in both peach varieties was higher than either glucose or fructose content (Fig. 2). In treated and untreated groups, as the sucrose content decreased, hexose (glucose and fructose) content generally increased. At harvest, the sucrose in 'Zajiao' peaches was $57.56 \mathrm{~g} / \mathrm{kg}$; 'Yulu' fruit contained $16.44 \%$ more sucrose with $67.02 \mathrm{~g} / \mathrm{kg}$. In the control peaches, by day 28 the sucrose content of 'Zajiao' and 'Yulu' peach fruit had fallen by $29.59 \%$ and $43.90 \%$ respectively, indicating that the rate of sucrose degradation was lower in 'Zajiao' than that of 'Yulu' during cold storage. 
Sucrose levels in both varieties of treated fruits were consistently higher than in control fruit, although the extent of this effect diminished after storage for $14 \mathrm{~d}$. 1-MCP treatment significantly $(p<0.01)$ increased the sucrose content in 'Zajiao' peaches during the first $7 \mathrm{~d}$, and significantly $(p<0.05)$ slowed the loss of sucrose in 'Yulu' peaches until day 28. In treated peaches the sucrose content decreased by $42.63 \%$ in 'Zajiao' peaches and $38.96 \%$ 'Yulu' peach fruit.

At harvest the glucose and fructose content in 'Zajiao' peach fruit was 8.21 and $4.14 \mathrm{~g} / \mathrm{kg}$ respectively and in 'Yulu' peach fruit, 3.60 and $2.15 \mathrm{~g} / \mathrm{kg}$ respectively. After $28 \mathrm{~d}$ of storage, glucose and fructose content in control 'Zajiao' peaches was 0.86 and 3.7 times that in control fruit respectively (Fig. 2B and C), while in control 'Yulu' peaches, levels increased 2 and 4.36 times respectively (Fig. 2E and F). These results indicate that fructose content increased faster than glucose content during cold storage, and that variation in the hexose content of 'Zajiao' peach fruit was less than in 'Yulu' peach fruit. The hexose content in 'Zajiao' peaches treated with 1-MCP was lower than that observed in controls, except for the fructose content at day 14 (Fig. 2B and C). In 'Yulu' peaches, there were no significant differences in hexose content between treated and control fruit before day 14, after day 21 glucose and fructose content in 1-MCP treated group was lower than in controls (Fig. 2E and F).

\subsection{Activities of AI, NI and SS-cleavage}

As shown in Fig. 3, the activities of several enzymes involved in sucrose catabolism were altered in 'Zajiao' and 'Yulu' peach fruit by the application of 1-MCP 
during postharvest cold storage. AI activity in 'Zajiao' and 'Yulu' control fruit increased up to day 21 and decreased subsequently. AI activity in 'Zajiao' peaches treated with 1-MCP was always significantly $(p<0.05)$ lower than that in the control group (Fig. 3A). Although AI activity in 1-MCP treated 'Yulu' peaches was significantly $(p<0.01)$ lower than that in control fruit after day 14 , there were no significant ( $p>0.05)$ differences before day 14 (Fig. 3D).

Although the profile of NI activity in treated and control 'Zajiao' peach fruit was the same, initially decreasing, then increasing until peaking at $28 \mathrm{~d}$ (Fig. 3B), the level of NI activity in 1-MCP-treated fruit was consistently lower than in control fruit. The NI activity in 'Yulu' peaches was relatively low during the first $14 \mathrm{~d}$ of storage, rapidly increased at day 21, and decreased thereafter (Fig. 3 E). Except at day 14, NI activity in1-MCP-treated 'Yulu' peach fruit was lower than in the control fruit. The pattern of SS-cleavage activity was similar to that observed for AI in 'Zajiao' peach fruit, and SS-cleavage activity in treated peaches was always lower than in control fruit (Fig. 3C). In contrast, for 'Yulu' peaches the trends and variations in SS-cleavage activity in control and treated fruit were nearly identical (Fig. 3F). Thus 1-MCP treatment effectively reduced the enzyme activities of AI, NI and SS-cleavage in 'Zajiao' peach fruit. In 'Yulu' peach fruit however, 1-MCP treatment only inhibited the rise of AI and NI activities late in cold storage, and had no effect on SS-cleavage activity.

\subsection{SS-synthesis and SPS activity}


As shown in Fig. 4A, SS-synthesis activity in 'Zajiao' peaches varied widely during storage. Except at day 7, activity in treated fruit was significantly $(p<0.01)$ higher than in the control fruit. SS-synthesis activity in treated 'Yulu' peaches was higher than in control fruit at day seven and thereafter, and peaked at day 14 (Fig. 4B). SPS activity in 'Yulu' and 'Zajiao' control samples peaked at day 14 but was always lower than observed in treated fruit (Fig. 4C and D). In 1-MCP treated groups, SPS activity initially increased and then decreased during cold storage, peaking at days 21 and 14 in 'Zajiao' and 'Yulu' peach fruit, respectively. The peak values in 'Zajiao' and 'Yulu' peach fruit treated with 1-MCP were 3.52 and 0.65 times that observed in control fruit, respectively.

\subsection{Transcription of genes involved in soluble sugar metabolism}

As shown in Fig. 5A and F, AI transcript levels increased rapidly early in storage, peaked at day 21, and then decreased in 'Zajiao' and 'Yulu' control and 1-MCP-treated fruit. The peak $A I$ levels were over one thousand times higher in 'Zajiao' fruit and hundreds of times higher in 'Yulu' fruit than levels observed at the beginning of the experiments.

The peak transcript levels for other genes tested (NI/1, NI/2, SS and SPS) were less than ten times the initial values, and were consistently relatively low (Fig. 5). NI/1 transcript levels in both varieties of 1-MCP-treated peaches were significantly $(p<0.01)$ lower than levels observed in controls, except at day 28 in 'Yulu' peach fruit.

(Fig. 5C and H). NI/2 transcript levels in treated 'Zajiao' peaches increased during the 
first $14 \mathrm{~d}$, then decreased and fell below control fruit on day 21 and 28 of cold storage. In contrast, 1-MCP-treatment inhibited the increase of NI/2 transcript levels in 'Yulu' peach fruit, and at no time during cold storage were the levels higher than the control fruit.

In 'Zajiao' peaches, untreated and 1-MCP-treated fruit displayed similar SS transcript profiles, increasing until day 21 and then decreasing. $S S$ transcript levels in fruit treated with 1 -MCP were significantly $(p<0.01)$ lower than in the controls (Fig. 5D). In 'Yulu' peaches, $S S$ transcript levels in control fruit were consistently higher than in 1-MCP-treatedfruit during cold storage (Fig. 5I).

Transcript levels of SPS peaked at day 7 and 14 in control and 1-MCP-treated 'Zajiao' peach fruit, respectively (Fig. 5E). Transcript levels in the treated peaches were significantly $(p<0.01)$ higher than in control fruit. In 'Yulu' peach fruit, control and 1-MCP-treated fruit displayed similar SPS transcription patterns, increasing until day 14 and then decreasing (Fig. 5J). SPS levels in control fruit were consistently lower than in treated fruit, and decreased to very low levels after day 21.

\section{Discussion}

CI symptoms in peach fruit are often observed within 1 or 2 weeks after storage at $2-5{ }^{\circ} \mathrm{C}$ (Lurie and Crisosto, 2005). Some varieties such as 'Jiubao' (Meng et al., 2009), and 'Yulu' (Wang et al., 2013) exhibit CI symptoms after $21 \mathrm{~d}$ at $5{ }^{\circ} \mathrm{C}$. 'Baifeng' (Jin et al., 2009) peach fruit exhibit CI symptoms after $21 \mathrm{~d}$ of storage at $0{ }^{\circ} \mathrm{C}$ plus 3 days of storage at $20{ }^{\circ} \mathrm{C}$. In our study, CI symptoms in 'Zajiao' peach fruit 
first manifested at day 28, while in 'Yulu' peach fruit they first occurred at day 21. The timing of CI is dependent on the peach variety, and 'Zajiao' fruit are more resistant to CI than 'Yulu' fruit.

1-MCP treatment appears to have a species-dependent effect on chilling tolerance. 1-MCP treatment inhibits CI in avocado (Pesis et al., 2002), persimmon (Salvador et al., 2004), plum (Candan et al., 2011), and loquat (Cao et al., 2010). On the other hand, CI severity is increased by 1-MCP treatment in banana (Jiang et al., 2004) and tomato (Zhang and Huang, 2010). In peach fruit, the effect of 1-MCP depends on variety and 1-MCP concentration. Jin et al. (2011) showed that $0.5 \mu \mathrm{L} / \mathrm{L}$ 1-MCP reduces CI development in 'Baifeng' peach fruit during cold storage by inhibiting $\mathrm{H}_{2} \mathrm{O}_{2}$ content and protecting membrane integrity. However, they also showed that $10 \mu \mathrm{L} / \mathrm{L} 1-\mathrm{MCP}$ increases the severity of CI. In 'Elberta' peach fruit, $\mathrm{CI}$ symptoms are aggravated by 1-MCP (Fan et al., 2002). Megías et al. (2016) proposed that 1-MCP exerts its effects by inhibiting ethylene signaling. In some plants, ethylene is a negative regulator of cold tolerance, and the inhibition of ethylene production by 1-MCP might plausibly reduce CI severity. In other species, ethylene seems to be an inducer of chilling tolerance since 1-MCP treatment reduces fruit quality. In our study, the $\mathrm{CI}$ severity was reduced by $1 \mu \mathrm{L} / \mathrm{L} 1-\mathrm{MCP}$ in both 'Zajiao' and 'Yulu' peach fruits (Fig. 1).

Soluble sugars may play an important role in protecting plants from chilling injury caused by oxidative stress (Couée et al., 2006). However, the evidence in support of this model is disputed. An early study reported that the loquat variety 
'Ninghaibai', which has higher resistance to low temperature, has higher levels of reducing sugars (including glucose and fructose) (Cao et al., 2013). Wang et al. (2016) showed that higher chilling tolerance in apricot fruit treated with oxalic acid was associated with higher a content of reducing sugars. However, in other plants, higher levels of sucrose are associated with enhanced chilling tolerance. In cucumber seedlings, exogenous sucrose pretreatment increases endogenous sucrose concentrations and alleviates chilling stress, apparently by regulating antioxidant enzyme activities (Cao et al., 2014). Wang et al. (2013) and Yu et al. (2015) have confirmed that higher sucrose content contributes to CI reduction in peach fruit during storage at $5{ }^{\circ} \mathrm{C}$. It has been proposed that methyl jasmonate treatment is effective in alleviating CI during cold storage by increasing the sucrose concentration (Yu et al., 2016). Heat treatment also has been shown to reduce CI in peach and mandarin fruit by enhancing the accumulation of sucrose (Holland et al., 2002; Yu et al., 2016). In our experiments, peach fruit treated with 1-MCP exhibited lower CI indexes and maintained higher levels of sucrose than untreated fruit during cold storage. Together, these results suggest that maintaining a higher content of sucrose protects peach fruit from CI during cold stress. The primary CI symptom observed in this report was browning, which results from membrane damage. Membrane lipid organization is changed by cellular dehydration caused by chilling stress, resulting in the loss of membrane semi-permeability (Couée et al., 2006; van den Bogaart et al., 2007). van den Bogaart et al. (2007) suggested that sucrose may combat leakage of cell membrane, because sucrose is a key molecule in determining 
the ability of plants to be cryopreserved (Couée et al., 2006). We found that 1-MCP treatment is beneficial for alleviating chilling stress in 'Zajiao' and 'Yulu' peach fruits because it inhibits the rate of sucrose degradation, resulting in a higher sucrose content during cold storage.

Interestingly, compared with 'Yulu' peach fruit, 'Zajiao' fruit has a lower sucrose content at harvest time, but exhibits higher resistance to chilling stress during storage. It indicated that higher content of sucrose at harvest time may not relate with the higher chilling resistance in peach fruits. However, it should be noticed that 'Yulu' fruit exhibited CI symptoms after $21 \mathrm{~d}$ of storage, during which the content of sucrose declined by $30 \%$. In contrast, sucrose loss in 'Zajiao' peaches was only $18.3 \%$ by day 21 and the fruit had no CI symptoms. Sucrose plays an important role as cryoprotectant in the plant (Couée et al., 2006; Folgado et al., 2016). Leaf osmotic potential increased significantly with the accumulation of sucrose upon petiole chilling of Amaranthus edulis L (Blechschmidt-Schneider et al., 1989). The increased accumulation of sucrose play a fundamental role in the response to stress in potato and be related to the enhancement in plant recovery after cryopreservation (Folgado et al., 2015; 2016). Higher levels of sucrose also contributed to membrane stability and enhanced chilling tolerance in peach fruit (Wang et al., 2013). On the contrary, it is obvious that the decreasing of sucrose content in plant will lose its role as cryoprotectant, which resulting in the destroy of membrane stability and the reduction of cold resistance. From the results of this study, it was suggested that the reduction in sucrose degradation, not the higher content of sucrose at harvest time, is 
essential for maintaining the membrane stability and chilling tolerance in peach fruits during the chilling stress. In fact, different postharvest treatments (Yu et al., 2016) or storage temperatures (Wang et al., 2013; Yu et al., 2015) that result in higher chilling tolerance, as well as 1-MCP treatment in the present study, all reduce the degradation of sucrose in peach fruits.

Degradation of starch in climacteric fruit is usually a source of sucrose, however, starch concentration in harvested peach fruit is very low and unlikely to contribute to sucrose changes (Bianco and Rieger, 2002; Yu et al., 2015). In sucrose metabolism, invertase (including AI and NI) and SS-cleavage are involved in transforming sucrose into glucose and fructose, while glucose and fructose are used as substrates to generate sucrose under the action of SS-synthesis and SPS (Cao et al., 2013). In order to understand more clearly which of these enzymes play important roles during cold storage, their activities have been investigated. Wang et al. (2016) reported that oxalic acid treatment of apricot enhanced the activities of AI, NI, SS-cleavage and SPS, and decreased the activity of SS-synthesis, leading to a reduction in sucrose content compared with untreated fruit. Cao et al. (2013) demonstrated that lower activities of SS-synthesis and SPS, and higher activities of AI, NI and SS-cleavage, contributed to the hydrolyzation of sucrose and the accumulation of reducing sugars in 'Ninghaibai' loquat fruit under chilling stress. In 'Feicheng' peach fruit treated with $10 \mu \mathrm{mol} / \mathrm{L}$ NO, SPS activity increased, and the activities of AI, NI and SS-cleavage decreased, resulting in the accumulation of sucrose (Sun et al., 2011). Yu et al. (2015) demonstrated that peach fruit stored at $5{ }^{\circ} \mathrm{C}$ had higher activities for all enzymes 
related to sucrose metabolism, but net cleavage activity was positive and increased during storage time, leading to reduced sucrose levels. In our study, 1-MCP treatment inhibited the activities of AI, NI, and SS-cleavage, and enhanced the activities of SS-synthesis and SPS, resulting in a net increase in sucrose content in 'Yulu' and 'Zajiao' peach fruit during cold storage.

In recent years, researchers have examined enzyme gene expression in order to investigate sucrose metabolism and the relationships between enzyme activities. Sucrose synthesis is determined by the activity and transcript levels of SPS (Nascimento et al., 1997). Transcript levels for three putative SPS genes (MrSPS1, MrSPS2 and MrSPS3) in Chinese bayberry fruit are markedly induced by blue light treatment, resulting higher sucrose levels during storage (Shi et al., 2016). MrINV transcription is also induced by blue light, but is significantly lower than observed for the MrSPS genes. This suggests that the SPS gene plays a more important role in sucrose synthesis than invertase. In cold plus 1-MCP treated Japanese pears, transcription of PpAIV1 and PpSPS1 is affected more than transcription of PpSUS1 and PpAIV2 (Itai and Tanahashi, 2008), suggesting that PpAIV1 and PpSPS1 expression are primarily responsible for sucrose metabolism during storage. Wang et al. (2013) suggest that the degradation of SPS/1 transcription is related to the decline in the sucrose level in peach fruit stored at $5{ }^{\circ} \mathrm{C}$. Interestingly, our previous studies demonstrate that $A I$ transcript levels initially increase sharply during storage at $5{ }^{\circ} \mathrm{C}$ before declining, with peak $A I$ levels as much as one thousand times higher than those observed in peach fruit immediately postharvest (Wang et al., 2013; Yu et al., 2015; 
Yu et al., 2016). The same trend was observed in the present study. AI transcript levels in control and 1-MCP-treated 'Zajiao' and 'Yulu' peach fruit increased dramatically, while NI/1-2, SS and SPS were expressed at relatively low levels (Fig 5). This demonstrates that $A I$ transcript levels are sensitive to chilling stress, and probably play the crucial role in modulating sucrose metabolism during cold storage. It is likely that higher SPS expression, combined with reduced $A I, N I / 1-2$ and $S S$ expression, is responsible for the higher content of sucrose in 'Zajiao' and 'Yulu' fruit treated with 1-MCP.

In summary, sucrose content decreases and the levels of reducing sugars increase in both varieties of peach fruits during cold storage. 'Zajiao' peach fruit exhibits higher chilling resistance than 'Yulu' peach fruit, which is consistent with the lower rate of sucrose degradation in 'Zajiao' peach fruit. 1-MCP treatment reduces the activity and expression of enzymes related to sucrose catabolism, and increases the activity and expression of enzymes associated with sucrose anabolism, reducing sucrose degradation 1-MCP treated fruit. We conclude that a lower sucrose degradation rate is related to higher chilling resistance in peach fruit.

\section{Acknowledgments}

This work was supported by the National Science Foundation of China (No. 31671903), the Nature Science Foundation of Zhejiang Province (No. LR15C200002), and the K. C. Wong Magna Fund at Ningbo University. 
Aubert, C., Bony, P., Chalot, G., Landry, P., Lurol, S., 2014. Effects of storage temperature, storage duration, and subsequent ripening on the physicochemical characteristics, volatile compounds, and phytochemicals of western red nectarine (Prunus persica L. Batsch). J. Agric. Food Chem. 62, 4707-4724.

Bianco, R.L., andRieger, M., 2002. Partitioning of sorbitol and sucrose catabolism within peach fruit. J. Am. Soc. Hort. Sci. 127, 115-121.

Blechschmidt-Schneider, S., Ferrar, P., Osmond, C.B., 1989. Control of photosynthesis by the carbohydrate level in leaves of the C4 plant Amaranthus edulis L. Planta 177,515-525.

Bolouri-Moghaddam, M.R., Le Roy, K., Xiang, L., Rolland, F., Van den Ende, W., 2010. Sugar signalling and antioxidant network connections in plant cells. FEBS J. 277, 2022-2037.

Candan, A.P., Graell, J., Larrigaudière, C., 2011. Postharvest quality and chilling injury of plums: benefits of 1-methylcyclopropene. Span. J. Agric. Res. 9, 554-564.

Cantín, C.M., Crisosto, C.H., Ogundiwin, E.A., Gradziel, T., Torrents, J., Moreno, M.A., Gogorcena, Y., 2010. chilling injury susceptibility in an intra-specific peach [Prunus persica (L.) Batsch] progeny. Postharvest Biol. Technol. 58, 79-87.

Cao, S., Yang, Z., Zheng, Y., 2013. Sugar metabolism in relation to chilling tolerance of loquat fruit. Food Chem. 136, 139-143.

Cao, S., Zheng, Y., Wang, K., Rui, H., Tang, S., 2009. Effects of 1-methylcyclopropene on oxidative damage, phospholipases and chilling injury in loquat fruit. J. Sci. Food Agr. 89, 2214-2220.

Cao, S., Zheng, Y., Wang, K., Rui, H., Tang, S., 2010. The effects of 1-methylcyclopropene on chilling and cell wall metabolism in loquat fruit. J. Hortic. Sci. Biotechnol. 85, 147-153.

Cao, Y., Yang, M., Li, X., Zhou, Z., Wang, X., Bai, J., 2014. Exogenous sucrose increases chilling tolerance in cucumber seedlings by modulating antioxidant enzyme activity and regulating proline and soluble sugar contents. Sci. Hortic. 179, 67-77.

Cáceres, D., Díaz, M., Shinya, P., Infante, R., 2016. Assessment of peach internal flesh browning through colorimetric measures. Postharvest Biol. Technol. 111, 48-52.

Cheng, S., Wei, B., Zhou, Q., Tan, D., Ji, S., 2015. 1-Methylcyclopropene alleviates chilling injury by regulating energy metabolism and fatty acid content in 'Nanguo' pears. Postharvest Biol. Technol. 109, 130-136

Couée, I., Sulmon, C., Gouesbet, G., El Amrani, A., 2006. Involvement of soluble sugars in reactive oxygen species balance and responses to oxidative stress in plants. J. Exp. Bot. 57, 449-459.

Dou, H., Jones, S., Ritenour, M., 2005. Influence of 1-MCP application and concentration on post-harvest peel disorders and incidence of decay in citrus fruit. J. Hortic. Sci. Biotechnol. 80, 786-792.

Fan, X., Argenta, L., Mattheis, J.P., 2002. Interactive effect of 1-MCP and temperature on 'Elberta' peach quality. Hort Sci. 37, 134-138.

Folgado, R., Panis, B., Sergeant, K., Renaut, J., Swennen, R., Hausman, J.F., 2015. Unravelling the effect of sucrose and cold pretreatment on cryopreservation of potato through sugar analysis and proteomics. Cryobiology 71, 432-441.

Folgado, R., Sergeant, K., Renaut, J., Swennen, R., Hausman, J., Panis, B., 2016. Changes in sugar content and proteome of potato in response to cold and dehydration stress and their implications for cryopreservation. J Proteomics 98, 99-111.

Giné-Bordonaba, J., Cantín, C.M., Echeverría, G., Ubach, D., Larrigaudière, C., 2016. The effect of chilling injury-inducing storage conditions on quality and consumer acceptance of different Prunus 
persica cultivars. Postharvest Biol. Technol. 115, 38-47.

Girardi, C.L., Corrent, A.R., Lucchetta, L., Zanuzo, M.R., Da Costa, T.S., Brachmann, A., Twymand, R.M., Nora, F.R., Nora, L., Silva, J.A., Romdaldi, C.V., 2005. Effect of ethylene, ntermittent warming and controlled atmosphere on postharvest quality and occurrence of wolliness in peach (Prunus persica cv. Chiripa) during cold storage. Postharvest Biol. Technol. 38, 25-33.

Holland, N., Menezes, H.C., Lafuente, M.T., 2002. Carbohydrates as related to the heat-induced chilling tolerance and respiratory rate of 'Fortune' mandarin fruit harvested at different maturity stages. Postharvest Biol. Technol. 25, 181-191.

Itai, A., Tanahashi, T., 2008. Inhibition of sucrose loss during cold storage in Japanese pear (Pyrus pyrifolia Nakai) by 1-MCP. Postharvest Biol. Technol. 48, 355-363.

Jiang, Y., Joyce, D.C., Jiang, W., Lu, W., 2004. Effects of chilling temperatures on ehtylene binding by banana fruit. Plant Growth Regul. 43, 109-115.

Jin, P., Shang, H., Chen, J., Zhu, H., Zhao, Y., Zheng, Y., 2011. Effect of 1-methylcyclopropene on chilling injury and quality of peach fruit during cold storage. J. Food Sci. 76, S485-S491.

Jin, P., Wang, K., Shang, H., Tong, J., Zheng, Y., 2009. Low-temperature conditioning combined with methyl jasmonate treatment reduces chilling injury of peach fruit. J. Sci. Food Agr. 89, 1690-1696.

Jin, P., Zhu, H., Wang, L., Shan, T., Zheng, Y., 2014. Oxalic acid alleviates chilling injury in peach fruit by regulating energy metabolism and fatty acid contents. Food Chem. 161, 87-93.

Livak, K.J., Schmittgen, T.D., 2001. Analysis of relative gene expression data using real-time quantitative PCR and the $2^{-\triangle \triangle C T}$. Methods. 25, 402-408.

Lurie, S., Crisosto, C.H., 2005. Chilling injury in peach and nectarine. Postharvest Biol. Technol. 37, 195-208.

Megías, Z., Martínez, C., Manzano, S., García, A., del Mar Rebolloso-Fuentes, M., Valenzuela, J.L., Garrido, D., Jamilena, M., 2016. Ethylene biosynthesis and signaling elements involved in chilling injury and other postharvest quality traits in the non-climacteric fruit of zucchini (Cucurbita pepo). Postharvest Biol. Technol. 113, 48-57.

Meisel, L., Fonseca, B., González, S., Baeza-Yates, R., Cambiazo, V., Campos, R., González, M., Orellana, A., Retamales, J., Silva, H., 2005. A rapid and efficient method for purifying high quality total RNA from peaches (Prunus persica) for functional genomics analyses. Biol. Res. 38, 83-88.

Meng, X., Han, J., Wang, Q., Tian, S., 2009. Changes in physiology and quality of peach fruits treated by methyl jasmonate under low temperature stress. Food Chem. 114, 1028-1035.

Nascimento, J.R.O., Cordenunsi, B.R., Lajolo, F.M., Alcocer, M.J.C., 1997. Banana sucrose-phosphate synthase gene expression during fruit ripening. Planta. 203, 283-288.

Özkaya, O., Yildirim, D., Dündar, Ö., Tükel, S.S., 2016. Effects of 1-methylcyclopropene (1-MCP) and modified atmosphere packaging on postharvest storage quality of nectarine fruit. Sci Hortic-Amsterdam. 198, 454-461.

Pesis, E., Ackerman, M., Ben-Arie, R., Feygenberg, O., Feng, X., Apelbaum, A., Goren, R., Prusky, D., 2002. Ethylene involvement in chilling injury symptoms of avocado during cold storage. Postharvest Biol. Technol. 24, 171-181.

Puig, P., Dagar, A., Ibanez, C.M., Singh, V., Crisosto, C.H., Friedman, H., Lurie, S., Granell, A., 2015. Pre-symptomatic transcriptome changes during cold storage of chilling sensitive and resistant peach cultivars to elucidate chilling injury mechanisms. BMC Genomics 16, 245.

Salvador, A., Arnal, L., Monterde, A., Cequerella, J., 2004. Reduction of chilling injury symptoms in persimmon fruit cv. 'Rojo Brillante' by 1-MCP. Postharvest Biol. Technol. 33, 285-291. 
Shao, X., Zhu, Y., Cao, S., Wang, H., Song, Y., 2013. Soluble sugar content and metabolism as related to the heat-induced chilling tolerance of loquat fruit during cold storage. Food and Bioprocess Technol. 6, 3490-3498.

Shi, L., Cao, S., Shao, J., Chen, W., Yang, Z., Zheng, Y., 2016. Chinese bayberry fruit treated with blue light after harvest exhibit enhanced sugar production and expression of cryptochrome genes. Postharvest Biol. Technol. 111, 197-204.

Solomakhin, A.A., Blanke, M.M., 2010. Mechanical flower thinning improves the fruit quality of aples. J. Sci. Food Agr. 35, 543-584.

Sun, Z., Li, Y., Zhou, J., Zhu, S.H., 2011. Effects of exogenous nitric oxide on contents of soluble sugars and related enzyme activities in 'Feicheng' peach fruit. J. Sci. Food Agric. 91, 1795-1800.

Thomsen, R., S $\varnothing$ Lvsten, C.A.E., Linnet, T.E., Blechingberg, J., Nielsen, A.L., 2010. Analysis of qPCR data by converting exponentially related $\mathrm{Ct}$ values into linearly related $\mathrm{XO}$ values. J. Bioinf. Comput. Biol. 8 , 885-900.

van den Bogaart, G., Hermans, N., Krasnikov, V., de Vries, A.H., Poolman, B., 2007. On the decrease in lateral mobility of phospholipids by sugars. Biophys. J. 92, 1598-1605.

Wang, K., Shao, X., Gong, Y., Xu, F., Wang, H., 2014. Effects of Postharvest Hot Air Treatment on Gene Expression Associated with Ascorbic Acid Metabolism in Peach Fruit. Plant Mol. Biol. Rep. 32, 881-887. Wang, K., Shao, X., Gong, Y., Zhu, Y., Wang, H., Zhang, X., Yu, D., Yu, F., Qiu, Z., Lu, H., 2013. The metabolism of soluble carbohydrates related to chilling injury in peach fruit exposed to cold stress. Postharvest Biol. Technol. 86, 53-61.

Wang, Z., Cao, J., Jiang, W., 2016. Changes in sugar metabolism caused by exogenous oxalic acid related to chilling tolerance of apricot fruit. Postharvest Biol. Technol. 114, 10-16.

Watkins, C.B., 2006. The use of 1-methylcyclopropene (1-MCP) on fruits and vegetables. Biotechnol Adv. 24, 389-409.

Yu, F., Ni, Z., Shao, X., Yu, L., Liu, H., Xu, F., Wang, H., 2015. Differences in sucrose metabolism in peach fruit stored at chilling stress versus nonchilling stress temperatures. Hort Sci. 50, 1542-1548.

Yu, L., Liu, H., Shao, X., Yu, F., Wei, Y., Ni, Z., Xu, F., Wang, H., 2016. Effects of hot air and methyl jasmonate treatment on the metabolism of soluble sugars in peach fruit during cold storage. Postharvest Biol. Technol. 113, 8-16.

Zhang, C., Shen, Z., Zhang, Y., Han, J., Ma, R., Korir, N.K., Yu, M., 2012. Cloning and expression of genes related to the sucrose-metabolizing enzymes and carbohydrate changes in peach. Acta Physiol. Plant. $35,589-602$.

Zhang, Z., Huang, R., 2010. Enhanced tolerance to freezing in tobacco and tomato overexpressing transcription factor TERF2/LeERF2 is modulated by ethylene biosynthesis. Plant mol. Biol. 73, 241-249. Zhou, H.W., Dong, L., Ben-Arie, R., Lurie, S., 2011. The role of ethylene in the prevention of chilling injury in nectarines. J Plant Physiol. 158, 55-61. 
Fig. 1. Effects of 1-MCP treatment on firmness and CI index in 'Zajiao' (A-B) and 'Yulu' (C-D) peach fruit stored at $5{ }^{\circ} \mathrm{C}$ for 35 and $28 \mathrm{~d}$, respectively. The values are expressed as means $\pm \mathrm{SE}$ of triplicate assays. ${ }^{*} p<0.05 ; * * p<0.01$ based on Duncan's multiple range test between the Control and 1-MCP treated groups at each time point.

Fig. 2. Effects of 1-MCP treatment on soluble sugar content in 'Zajiao' (A-C) and 'Yulu' (D-F) peach fruit stored at $5{ }^{\circ} \mathrm{C}$ for 35 and $28 \mathrm{~d}$, respectively. The values are expressed as means $\pm \mathrm{SE}$ of triplicate assays. $* p<0.05 ; * * p \quad 0.01$ based on Duncan's multiple range test between the Control and 1-MCP treated groups at each time point.

Fig.3. Effects of 1-MCP treatment on AI (A), NI (B) and SS-cleavage (C) activities in 'Zajiao' fruit, and AI (D), NI (E) and SS-cleavage (F) activities in 'Yulu' fruit stored at $5{ }^{\circ} \mathrm{C}$ for 35 ('Zajiao') and 28 ('Yulu') d. The values are expressed as means $\pm \mathrm{SE}$ of triplicate assays. $* p<0.05 ; * * p<0.01$ based on Duncan's multiple range test between the Control and 1-MCP treated groups at each time point.

Fig.4. Effects of 1-MCP treatment on SS-synthesis (A) and SPS (B) activities in 'Zajiao' and SS-synthesis (C) and SPS (D) activities in 'Yulu' peach fruit stored at $5{ }^{\circ} \mathrm{C}$ for 35 ('Zajiao') and 28 ('Yulu') d. The values are expressed as means $\pm \mathrm{SE}$ of triplicate assays. ${ }^{*} p<0.05 ; * * p<0.01$ based on Duncan's multiple range test 
between the Control and 1-MCP treated groups at each time point.

Fig. 5. Effects of 1-MCP treatment on relative transcript levels of genes involved in sucrose metabolism in 'Zajiao' (A-E) and 'Yulu' (F-J) peach fruit stored at $5{ }^{\circ} \mathrm{C}$ for 35 and $28 \mathrm{~d}$ respectively. The values are expressed as means $\pm \mathrm{SE}$ of triplicate assays. ${ }^{*} p$ $<0.05$; ** $p<0.01$ based on Duncan's multiple range test between the Control and 1-MCP treated groups at each time point. 

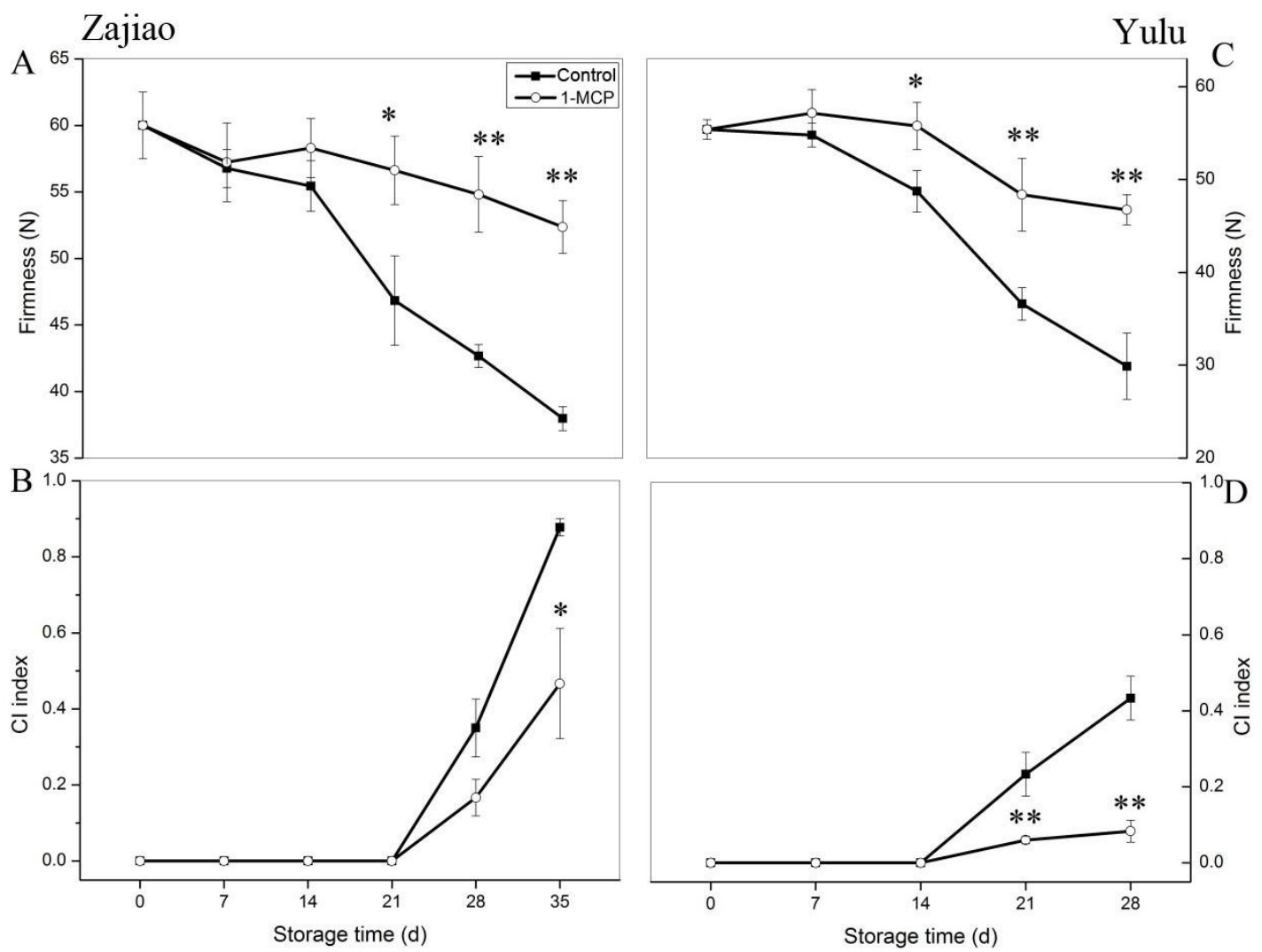

Fig.1 

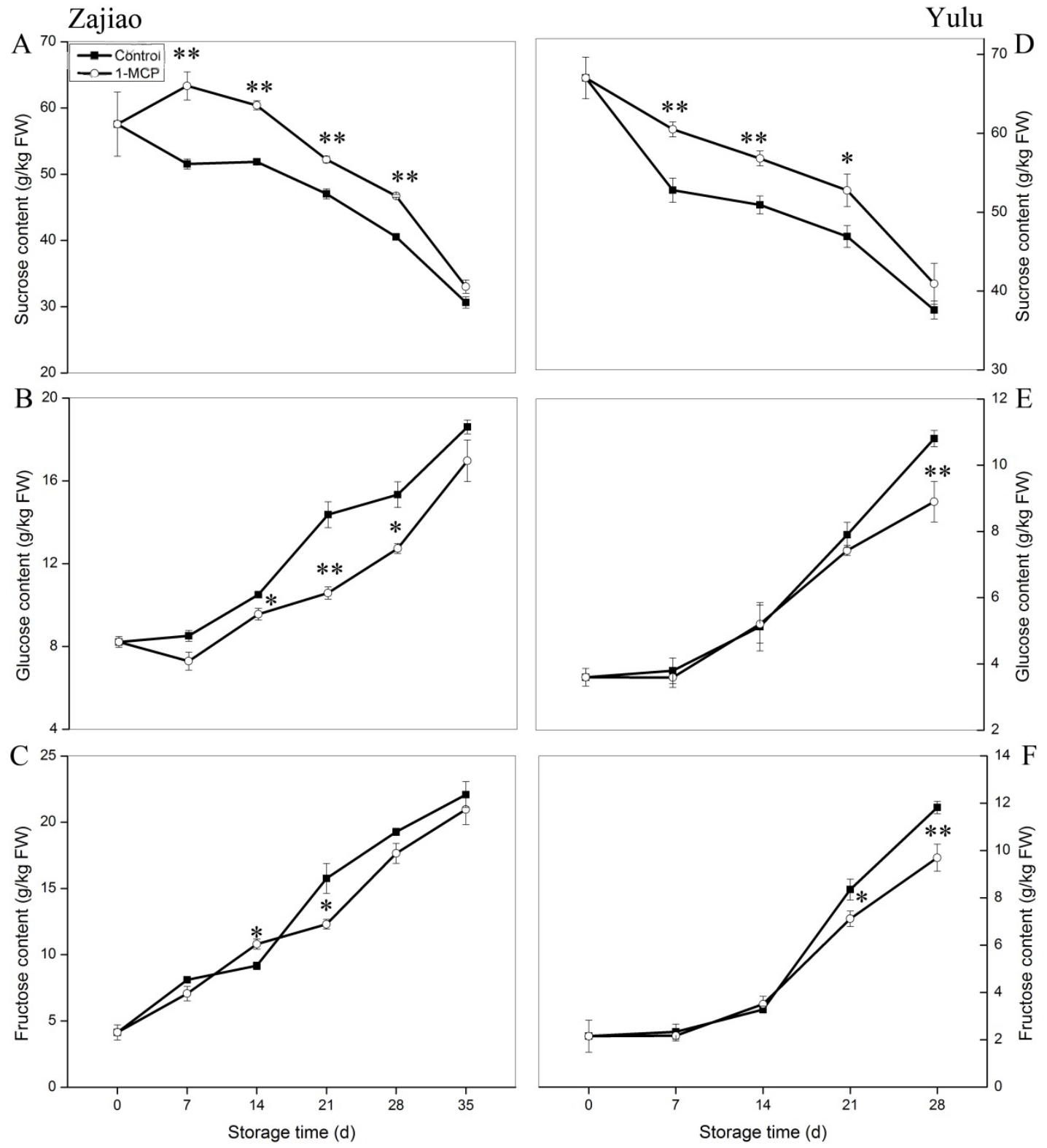

Fig.2 
Zajiao
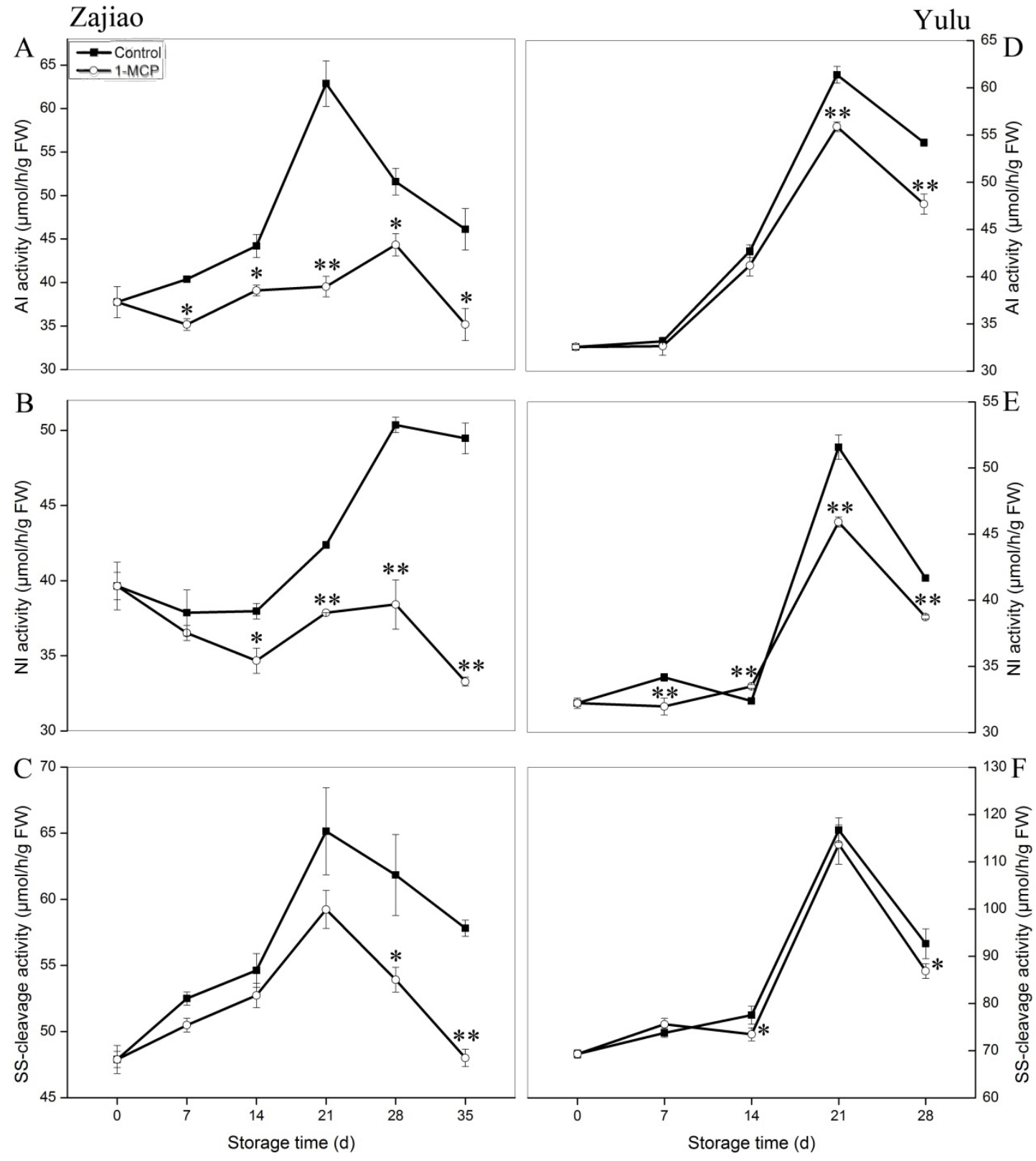

Fig.3 

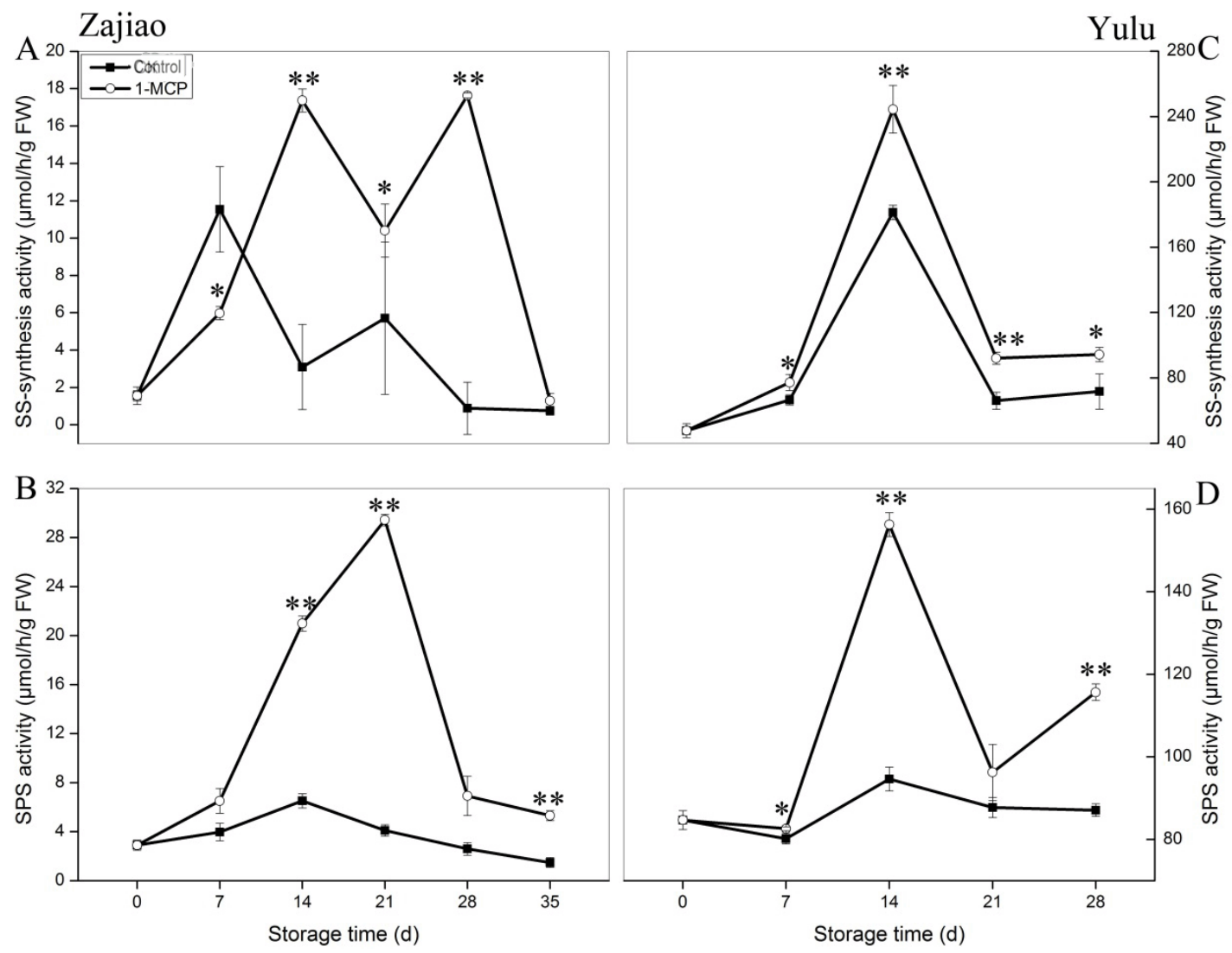

Fig.4 


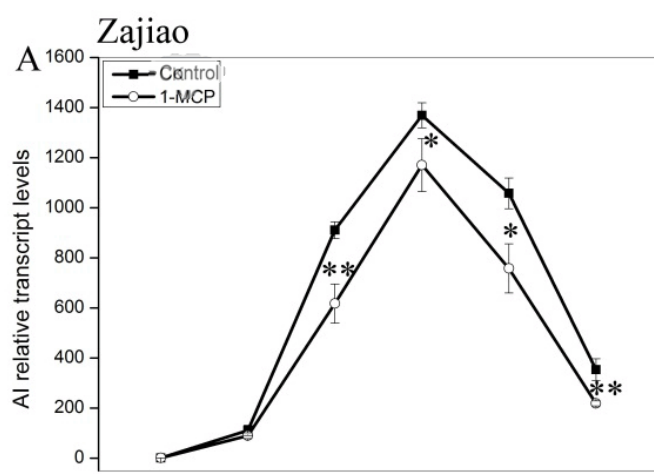

Yulu
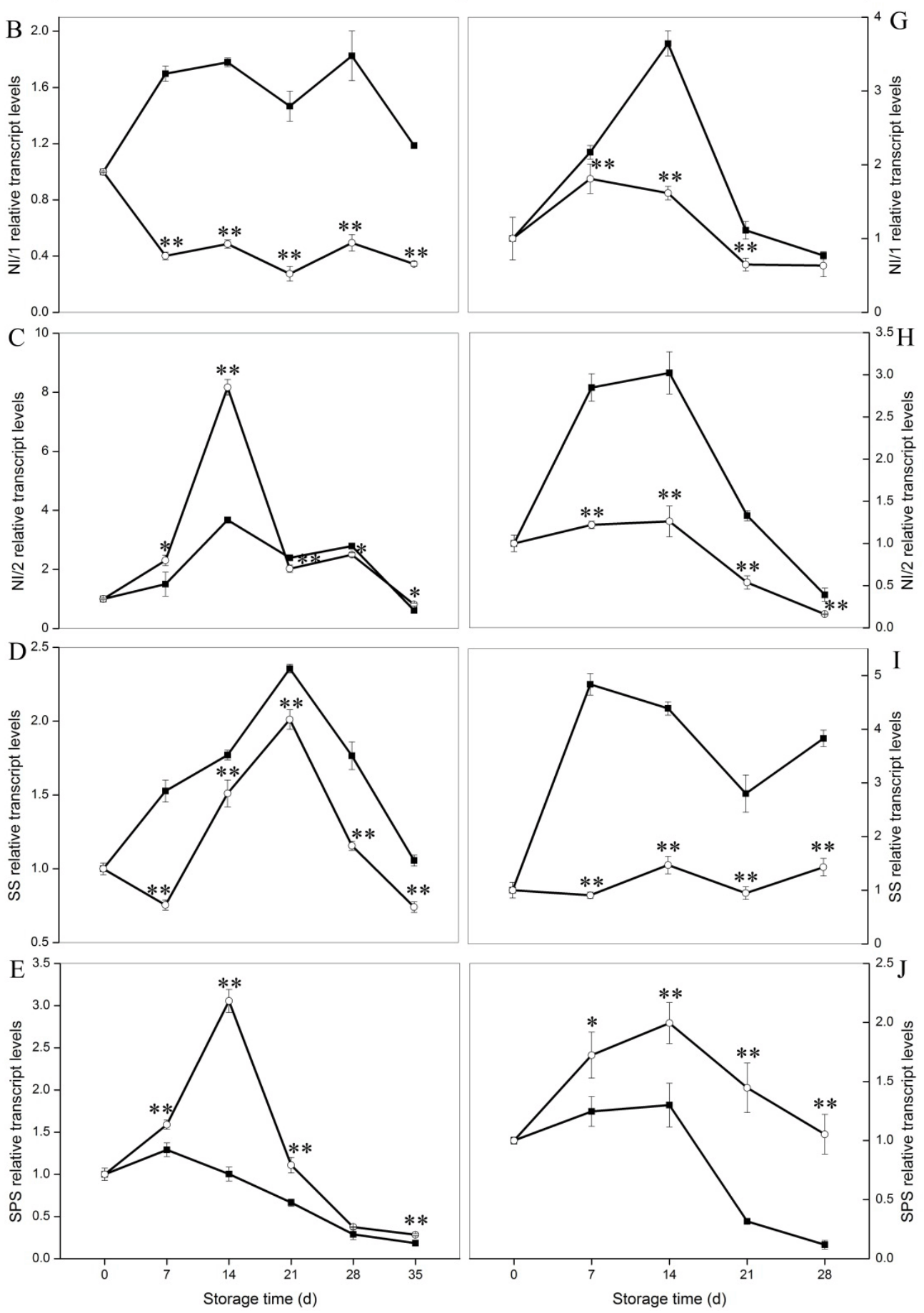

Fig.5 